\title{
Wenzel, Hartmut
}

\section{Lernwerkstätten auch für die Sekundarstufen. Zur Entwicklung der}

\section{Hochschullernwerkstatt in Halle}

Kramer, Kathrin [Hrsg.]; Rumpf, Dietlinde [Hrsg.]; Schöps, Miriam [Hrsg.]; Winter, Stephanie [Hrsg.]: Hochschullernwerkstätten - Elemente von Hochschulentwicklung? Ein Rückblick auf 15 Jahre Hochschullernwerkstatt in Halle und andernorts. Bad Heilbrunn : Verlag Julius Klinkhardt 2020, S. 45-54. - (Lernen und Studieren in Lernwerkstätten)

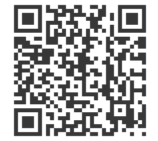

Quellenangabe/ Reference:

Wenzel, Hartmut: Lernwerkstätten auch für die Sekundarstufen. Zur Entwicklung der

Hochschullernwerkstatt in Halle - In: Kramer, Kathrin [Hrsg.]; Rumpf, Dietlinde [Hrsg.]; Schöps, Miriam [Hrsg.]; Winter, Stephanie [Hrsg.]: Hochschullernwerkstätten - Elemente von Hochschulentwicklung? Ein Rückblick auf 15 Jahre Hochschullernwerkstatt in Halle und andernorts. Bad Heilbrunn : Verlag Julius Klinkhardt 2020, S. 45-54 - URN: urn:nbn:de:0111-pedocs-212014 - DOI: 10.25656/01:21201

https://nbn-resolving.org/urn:nbn:de:0111-pedocs-212014

https://doi.org/10.25656/01:21201

in Kooperation mit / in cooperation with:

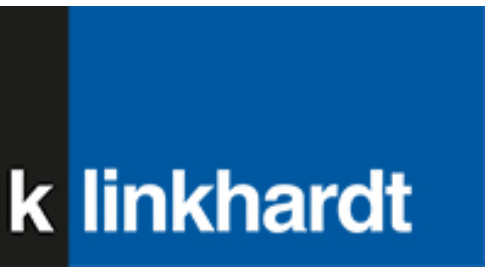

http://www.klinkhardt.de

\section{Nutzungsbedingungen}

Dieses Dokument steht unter folgender Creative Commons-Lizenz: http://creativecommons.org/licenses/by-nc-sa/4.0/deed.de - Sie dürfen das Werk bzw. den Inhalt unter folgenden Bedingungen vervielfältigen, verbreiten und öffentlich zugänglich machen sowie Abwandlungen und Bearbeitungen des Werkes bzw. Inhaltes anfertigen: Sie müssen den Namen des Autors/Rechteinhabers in der von ihm festgelegten Weise nennen. Dieses Werk bzw. der Inhalt darf nicht für kommerzielle Zwecke verwendet werden. Die neu entstandenen Werke bzw. Inhalte dürfen nur unter Verwendung von Lizenzbedingungen weitergegeben werden, die mit denen dieses Lizenzvertrages identisch oder vergleichbar sind.

Mit der Verwendung dieses Dokuments erkennen Sie die Nutzungsbedingungen an.

\section{Terms of use}

This document is published under following Creative Commons-License: http://creativecommons.org/licenses/by-nc-sa/4.0/deed.en - You may copy, distribute and transmit, adapt or exhibit the work in the public and alter, transform or change this work as long as you attribute the work in the manner specified by the author or licensor. You are not allowed to make commercial use of the work. If you alter, transform, or change this work in any way, you may distribute the resulting work only under this or a comparable license.

By using this particular document, you accept the above-stated conditions of use.

\section{Kontakt / Contact:}

peDOcs

DIPF | Leibniz-Institut für Bildungsforschung und Bildungsinformation

Informationszentrum (IZ) Bildung

E-Mail: pedocs@dipf.de

Internet: www.pedocs.de

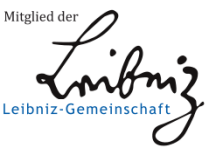




\section{Lernen und Studieren in Lernwerkstätten}

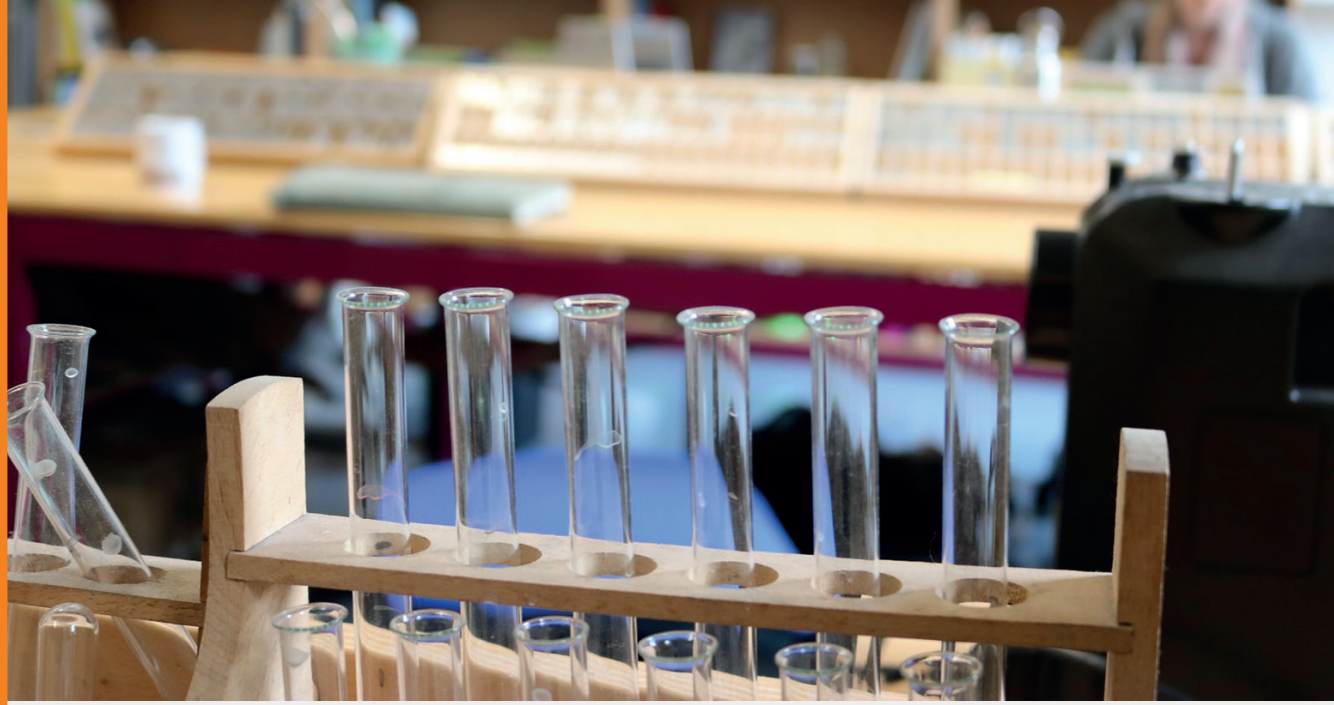

Kathrin Kramer / Dietlinde Rumpf / Miriam Schöps / Stephanie Winter (Hrsg.)

Hochschullernwerkstätten - Elemente von Hochschulentwicklung?

Ein Rückblick auf 15 Jahre Hochschullernwerkstatt in Halle und andernorts 


\section{Kramer / Rumpf / Schöps / Winter Hochschullernwerkstätten - Elemente von Hochschulentwicklung?}




\section{Lernen und Studieren in Lernwerkstätten}

Impulse für Theorie und Praxis

Herausgegeben von

Eva-Kristina Franz, Johannes Gunzenreiner, Barbara Müller-Naendrup, Hartmut Wedekind und Markus Peschel 


\section{Kathrin Kramer \\ Dietlinde Rumpf \\ Miriam Schöps \\ Stephanie Winter \\ (Hrsg.)}

\section{Hochschullernwerkstätten - Elemente von Hochschulentwicklung?}

Ein Rückblick auf 15 Jahre Hochschullernwerkstatt in Halle und andernorts 
Der vorliegende Band ist anlässlich einer Tagung des 15jährigen Bestehens der Hochschullernwerkstatt Erziehungswissenschaften in Halle im November 2020 entstanden.

Gefördert wurden die Tagung und der Band vom Zentrum für Lehrer *innenbildung, der Universitäts- und Landesbibliothek Sachsen-Anhalt sowie der Martin-Luther-Universität Halle-Wittenberg.

Dieser Titel wurde in das Programm des Verlages mittels eines Peer-Review-Verfahrens aufgenommen. Für weitere Informationen siehe www.klinkhardt.de.

Bibliografische Information der Deutschen Nationalbibliothek Die Deutsche Nationalbibliothek verzeichnet diese Publikation in der Deutschen Nationalbibliografie; detaillierte bibliografische Daten sind im Internet abrufbar über http://dnb.d-nb.de.

2020.k. (C) by Julius Klinkhardt.

Coverfoto: (C) Florian Johnke-Liese / Hochschullernwerkstatt.

Druck und Bindung: AZ Druck und Datentechnik, Kempten.

Printed in Germany 2020.

Gedruckt auf chlorfrei gebleichtem alterungsbeständigem Papier.

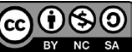

Die Publikation (mit Ausnahme aller Fotos, Grafiken und Abbildungen) ist veröffentlicht unter der Creative Commons-Lizenz: CC BY-NC-SA 4.0 International https://creativecommons.org/licenses/by-nc-sa/4.0/ 


\section{Inhalt}

Kathrin Kramer, Dietlinde Rumpf, Miriam Schöps und Stephanie Winter Einführung in den Band

\section{Teil 1: Historische Entwicklung}

Miriam Schöps und Dietlinde Rumpf

Universität mit Lernwerkstatt in Entwicklung -

Von der Materialausleihe zur Hochschuldidaktik

Hartmut Wenzel

Lernwerkstätten auch für die Sekundarstufen -

Zur Entwicklung der Hochschullernwerkstatt in Halle

Ulrike Stadler-Altmann, Susanne Schumacher, Enrico A. Emili,

Gerda Winkler und Elisabeth Dalla Torre

Hochschullernwerkstätten als Spielball der Bildungspolitik?

Die EduSpace Lernwerkstatt in der Südtiroler Lehrer*innenbildung zwischen nationalen und regionalen Bildungsinteressen

Hartmut Wedekind und Corinna Schmude

Von der Idee zum strukturell verankerten hochschuldidaktischen Prinzip -

Lernwerkstätten und Lernwerkstattarbeit im Studiengang „Erziehung und Bildung in der Kindheit" an der Alice Salomon Hochschule Berlin 68

\section{Teil 2: Wahrnehmung, Selbstverständnis, Einflüsse}

Dietlinde Rumpf und Corinna Schmude

NeHle - Internationales Netzwerk der Hochschullernwerkstätten -

Entwicklungsphasen einer Interessenvertretung und

eines gemeinsamen Begriffsverständnisses

Constantin Beyer und Florian Johnke-Liese

Hochschullernwerkstatt goes digital!

Alexandra und Michael Ritter

Drucken wie ,von gestern' in der Schule der Zukunft -

Die Schuldruckerei als Erfahrungsraum für Kinder und Studierende 
Livia Enders (geb. Makrinus) und Stephanie Winter

Studierende und Dozierende im Spannungsfeld zwischen

Theorie und Praxis - Die Hochschullernwerkstatt als Forschungsraum

John Marcus Sommer

Selbstwahrnehmung in der Hochschullernwerkstatt -

Wissenschaft zwischen Abstraktion und emotionalem Lernen

Lena Kliebe

Hochschullernwerkstatt als Raum und pädagogische Konzeption -

„Mein eigener Lernprozess“

Jerome Kampe

Geschichtsunterricht in der Lernwerkstatt!?

\section{Teil 3: Partizipation und Inklusion}

Melanie Schlag

Hochschullernwerkstatt schlägt Schlager

Kathrin Kramer

Nichtreformistische Reformen - Die Bedeutung von

Hochschullernwerkstätten auf dem Weg zu einer inklusiven Universität am Beispiel des Budgets für Arbeit

Johanna Ingenerf und Pascal Kurz

Menschenrechtsbasierte Bildung im internationalen Dialog -

Ein selbstorganisiertes Seminar mit Austausch

Ines Boban und Andreas Hinz

Hochschullernwerkstatt Halle als Möglichkeitsort -

Von WIRkstatt-Impulsen für inklusive Prozesse

Brigitte Kottmann und Alena Lensker (geb. Beckmann)

Die Lernwerkstatt und der Studiengang „Integrierte Sonderpädagogik“

an der Universität Bielefeld: Impulse, Synergien und Reflexionen

Sarah Dannemann, Tjark Neugebauer, Claudia Schomaker und Rolf Werning

Die LeibnizLernlandschaft: Diversität und Digitalisierung $\left(\mathrm{L}^{2} \mathrm{D}^{2}\right)$

gestalten - Konzeptionelle Gedanken für eine inklusive

Hochschullernwerkstatt an der Leibniz Universität Hannover 


\section{Teil 4: Reflexivität und Professionalisierung}

Kira Wybierek

Praxissemester in der Hochschullernwerkstatt -

Reflexion eines Lernprozesses

Eva Hoffart

„...da man lernt, eigene Gedanken und Ideen noch einmal zu vertiefen“ -

Theoretische Überlegungen und praktische Umsetzungen zum Reflektieren

von Lehramtsstudierenden

Edita Jung und Lena S. Kaiser

„Vielleicht romantisiere ich die Räume meiner Kindheit.“

Zugänge zu einer biographischen Reflexivität in

kindheitspädagogischen Hochschullernwerkstätten

Rolf-Torsten Kramer

Zum Problem der Professionalisierung im Lehramtsstudium und

zum Potenzial der Hochschullernwerkstatt

Mark Weißhaupt, Kathleen Panitz und Elke Hildebrandt

Die Inszenierung von „Theorie und Praxis“ sowie

„Neugier und Desinteresse“ bei der Professionalisierung

angehender Lehrpersonen in Hochschullernwerkstätten

Markus Peschel und Pascal Kihm

Hochschullernwerkstätten - Rollen, Rollenverständnisse und

Rollenaushandlungen

David Paulus, Patrick Gollub und Marcel Veber

Forschendes Lernen und Kasuistik: Überschneidungen und

Abgrenzungen bezogen auf Reflexivität in der

Hochschullernwerkstattarbeit

Pascal Kihm, Jenny Diener und Markus Peschel

Qualifizierungsprozesse und Qualifikationsarbeiten in

Hochschullernwerkstätten - Forschende Entwicklung einer

innovativen Didaktik 
10| Inhaltsverzeichnis

\section{Teil 5: Kooperation}

Georg Breidenstein, Sara Burkhardt, Thorid Rabe und Miriam Schöps

Zur Materialität des Lernens -

Anregungen aus einem interdisziplinären Forum

in der Hochschullernwerkstatt

Miriam Asmus, Kati Friebe, Mirjam Lewin und Kati Misselwitz

Entdeckendes Lernen und Digitale Medien - Ein Traumpaar .

Siglinde Spuller

Kooperation und Kooperatives Lernen als Prinzip

Hochschullernwerkstätten-adäquaten Lernens?

Eine konzeptionelle Verortung

Linda Balzer

Der Effekt selbstregulierenden und forschenden Lernens auf kooperative und individuelle Lernprozesse in der

Lernwerkstatt Religion Plural (LeRP)

Martin Lindner

Werkstattarbeit in der Biologiedidaktik -

Umstellung eines Praktikums auf Projektarbeit

Autor*innenbeschreibungen 
Hartmut Wenzel

\section{Lernwerkstätten auch für die Sekundarstufen - Zur Entwicklung der Hochschullernwerkstatt in Halle}

\section{Zusammenfassung}

Der Beitrag geht auf die Entstehungsgeschichte der halleschen Hochschullernwerkstatt ein. Er arbeitet dabei die drei unterschiedlichen Wurzeln heraus und schildert dann den bildungstheoretischen und schulpädagogischen Diskussionszusammenhang, von dem aus generell Lernwerkstätten in den 1990er Jahren und speziell diejenige in Halle für die Sekundarstufen eingerichtet wurde. Deutlich wird dabei, dass die Gründe, die zur Einrichtung einer Hochschullernwerkstatt für die Sekundarstufen führten, noch immer aktuell sind.

\section{Einleitung}

Die jetzige Hochschullernwerkstatt der Philosophischen Fakultät III Erziehungswissenschaft der Martin-Luther-Universität im Haus 31 der Franckeschen Stiftungen hat (mindestens) drei Wurzeln:

1. die Hochschullernwerkstätten aus dem Grundschulbereich, die bereits 1993 in Köthen begonnen wurden und mit der Übersiedlung des Instituts für Grundschulpädagogik 1997 aus Köthen nach Halle kamen;

2. die Hochschullernwerkstatt aus dem Institut für Pädagogik, die ab 1993/1994 von Meinert Meyer noch am Standort Brandbergweg begonnen und im Wintersemester 1996 in die Franckeschen Stiftungen verlegt wurde;

3. die Montessori-Werkstatt des Instituts für Rehabilitationspädagogik, die noch in den Institutsräumen an der Selkestraße begonnen und zusammen mit dem Institut für Rehabilitationspädagogik 2005 in die Franckeschen Stiftungen in die jetzigen Räumlichkeiten verlegt wurde.

Alle drei Vorläufereinrichtungen wurden 2005 nach der Rekonstruktion des jetzigen Hauses 31 (Hans-Ahrbeck-Haus) in den derzeitigen Räumen untergebracht. Das Raumkonzept der Hochschullernwerkstatt wurde in Kooperation mit 
dem Architekturbüro Ziegemeyer von Vertreter*innen der drei Institute ${ }^{1}$ gemeinsam entwickelt und führte zu der jetzigen Hochschullernwerkstatt, die neben Studierenden aller Lehrämter auch Interessierte der Uni, aber auch die anderer Institutionen einlädt.

Dass in drei unterschiedlichen pädagogischen Instituten nahezu gleichzeitig Lernwerkstätten begonnen wurden, belegt, dass damals die allgemeindidaktische, die schulpädagogische, aber auch die lerntheoretische Diskussion auf die verstärkte Nutzung von aktivierenden Lern- und Arbeitsformen in der Schule, die über den Frontalunterricht hinausgehen, drängten.

Im Folgenden sollen wesentliche Aspekte der bildungstheoretischen und schulpädagogischen Diskussion und damit Begründungen für die Einrichtung von Lernwerkstätten besonders für die Sekundarstufen angeführt werden, die mit dazu beitrugen, dass Mitte der 1990er Jahre eine Hochschullernwerkstatt mit diesem Schwerpunkt eingerichtet wurde.

\section{Zur Einrichtung der Lernwerkstatt am Institut für Pädagogik in Halle}

Die Einrichtung und Nutzung der Lernwerkstatt des Instituts für Pädagogik wurde nach der Berufung von Prof. Dr. Meinert Meyer (Professur für Allgemeine Didaktik/Schulforschung) zum Wintersemester 1993/94 in den Räumen der früheren Beratungsstelle des Fachbereichs Pädagogik am Brandbergweg begonnen. Meinert Meyer hatte in seinem Berufungsverfahren Mittel für die Einrichtung einer Hochschullernwerkstatt verhandelt und zugesagt bekommen.

In einem Interview antwortete Meyer auf die Frage: Warum wollten Sie eine Lernwerkstatt in Halle an der Uni aufbauen?

"Ich habe einen Bericht über die Laborschule in Chicago gelesen, die von John Dewey 1896 eingerichtet und 7 oder 8 Jahre betrieben wurde, in der ein völlig anderes Lernen versucht wird. John Dewey ging davon aus, dass man am besten dadurch lernt, dass man es tut, "learning by doing“. Dann ist mir bei der Lehrerfortbildung klar geworden, dass Schulzimmer doch das Ödeste sind, was man sich denken kann. Ein leerer Raum mit Tischen, Bänken, Lehrerpult und, wenn es hoch kommt, ein paar Bilder an der Wand. Dann hat sich allmählich das entwickelt, man müsste doch was machen. Ich habe dann zuerst mit Lehrern in Münster kooperiert, für eine Fremdsprachenwerkstatt. Also die gleiche Idee aber für Gymnasien, und ausgelegt für Fremdsprachen. Als ich dann den Ruffür Halle bekommen habe, war das gleich eine der Sachen, die ich aushandeln wollte. Und das ging erstaunlich problemlos" (MEYER 1996).

1 Das jetzige Institut für Schulpädagogik und Grundschuldidaktik wurde erst im Jahr 2000 gegründet. 
Da Mitte der 1990er Jahre die Zahl der Lehramtsstudent*innen massiv eingebrochen war, öffnete Meinert Meyer seine Lehrveranstaltungen zu Lernwerkstätten auch für Student*innen des Studienganges Diplom Erziehungswissenschaft. Nach dem Weggang von Meinert Meyer (Berufung nach Hamburg zum Wintersemester 1996/97) übernahm vor allem Dr. Rolf Luther die Leitung dieser Hochschullernwerkstatt, bezüglich der Sekundarschulschwerpunkte unterstützt von mir selbst. Nach Übersiedlung des Instituts für Grundschulpädagogik wurde diese Hochschullernwerkstatt auch für die Grundschulpädagogik (vor allem Deutsch, Sachunterricht) genutzt. Während des Umbaus von Haus 31 lagerte das Inventar ein und wurde später in die Hochschullernwerkstatt am jetzigen Standort in Haus 31 eingebracht.

\section{Warum Lernwerkstätten für die Sekundarstufen I und II?}

1968 wurde in den alten Bundesländern eine eigenständige Grundschule für die Jahrgänge 1-4 eingerichtet. Die Jahrgänge 5-8 und später auch 9 und sogar 10 wurden getrennt davon in der neu eingerichteten Hauptschule zusammengefasst, parallel zu Realschule und Gymnasium. Seither kam es zuerst in den Grundschulen und dann langsam auch in den weiteren Schulstufen zu einer Renaissance reformpädagogischer Ansätze, die in der Nazi- und direkten Nachkriegszeit verboten bzw. verdrängt waren. Zu diesen gehörten neben der Waldorf-, Montessori- und Jenaplan-Pädagogik im Laufe der 1970er Jahre auch die Freinet-Pädagogik sowie der Projektunterricht nach Dewey und Kilpatrick. Diese für eine schüler*innenorientierte Praxis relevanten Ansätze fanden Aufnahme nicht nur in reformorientierten Schulen, sondern in den 1970er Jahren langsam auch in die universitäre schulpädagogische Diskussion im Rahmen der Lehrer*innenbildung. Eine vergleichbare Hinwendung zu reformpädagogischen Ansätzen hat es in der DDR nicht gegeben.

Ich selbst habe mich bereits seit Ende der 1960er Jahre im Rahmen des Studiums und der studentischen Fachschaftsarbeit mit Fragen der Studien- und Unterrichtsreform beschäftigt. Ich hatte dann während meiner Assistentenzeit an der Universität Essen die Gelegenheit, an einem mehrjährigen Forschungsprojekt zum „schüleraktiven Unterricht" (BoHNSACK et al. 1984) mitzuarbeiten. Ziel des Forschungsprojektes war es, die damalige Diskussion zu Krisenphänomenen der Schule mit dem Schwerpunkt "Schulmüdigkeit“ aufzunehmen und Schulmüdigkeit durch Formen „schüleraktiven Unterrichts" zu überwinden. Wir versuchten also, Lehrer*innengruppen an unterschiedlichen Schulen der Sekundarstufen (Gymnasium, Hauptschule) dazu zu bewegen, verstärkt einen „schülerorientierten“, „schülerzentrierten “oder „offenen “Unterricht zu praktizieren. Die zentrale 
Frage des Projektes lautete damals: weshalb haben die bereits länger vorliegenden und vielfach erfolgreich erprobten reformorientierten Ansätze den Alltagsunterricht kaum verändert, wo liegen die Barrieren und welche Spielräume für Veränderungen bestehen heute (BoHNSACK et al. 1984, 1)? Eines der bedeutsamen Ergebnisse des damaligen Projektes bestand darin, dass es in der Schule durchaus Gestaltungsmöglichkeiten für einen „schüleraktiven Unterricht“ gibt, wenn auch die Barrieren, die durch organisatorische Regelungen des schulischen Alltags erlebt werden, insbesondere durch die Benotungs- und Versetzungspraxis, aber auch der Stundenplangestaltung, ganz erheblich sind. Die Barrieren lassen sich verringern, wenn in einem Kollegium gemeinsam an Veränderungen gearbeitet wird. Solche Erfahrungen sind später in Ansätze der Schulinternen Lehrer*innenfortbildung (WenZel, WesEMANn \& Bohnsack 1990) und der Schulprogrammarbeit eingeflossen.

In meiner Habilitationsschrift „Unterricht und Schüleraktivität“ (Wenzel 1987) habe ich mich dann - aufbauend auf den Erfahrungen des genannten Projektes - mit Problemen und Möglichkeiten der Entwicklung von Selbststeuerungsfähigkeiten im Unterricht auseinandergesetzt. In diesem Zusammenhang habe ich auch intensiv die Diskussion um den Handlungsorientierten Unterricht verfolgt. Handlungsorientierter Unterricht wurde in den pädagogischen Medien etwa ab 1980 zu einem viel beachteten Konzept, führte zu vielfältigen fachdidaktischen Unterrichtsbeispielen, zu einschlägigen Wettbewerben und zahlreichen Publikationen (z. B. Frey 1982; Gudjons 1987). Befördert wurden diese unterrichtsbezogenen Reformbemühungen durch die lerntheoretischen Diskussionen in der Nachfolge von Piaget und des Konstruktivismus. Betont wurde in diesem Zusammenhang, dass Lernen ein aktiver, personeninterner Prozess ist. Diese Erkenntnisse sollten natürlich Konsequenzen für die Unterrichtsgestaltung haben, die Kritik an frontalunterrichtlichen Verfahren wuchs. In dieser Zeit richteten fortschrittlichere, reformpädagogisch inspirierte Schulen bereits in Anlehnung an Maria Montessori, Rudolf Steiner, Peter Petersen und Célestin Freinet Lernwerkstätten ein, denen sich bald Überlegungen anschlossen, auch in der Lehrer*innenbildung Möglichkeiten zu schaffen, aktivierende Unterrichtsformen bereits im Studium kennenzulernen und so auch auf projektartiges Arbeiten und die Arbeit in schulischen Lernwerkstätten vorzubereiten.

Bedeutsame neue Anstöße für ein verändertes Verständnis vom Lernen ergaben sich zu jener Zeit drängend aus dem „Lernbericht" des Club of Rome (PeCCEI 1980). Anknüpfend an dessen 1972 veröffentlichten Bericht über die „Grenzen des Wachstums" (Meadows 1972) wurde nun argumentiert, dass die Menschheit an einem bedeutsamen Scheideweg angelangt sei, vor einem „menschlichen Dilemma“ stehe. Die Menschheit habe Voraussetzungen geschaffen, dass es zukünftig allen Menschen erheblich besser gehen könnte, aber zugleich die Möglichkeiten - etwa im Hinblick auf Atomwaffen und -technik, aber auch Natur- 
und Umweltbelastung -, das menschliche Leben auf dieser Erde zu vernichten. Es liege nun - so die aufstörende These - an der Menschheit selbst, zukünftige Entscheidungen so zu treffen, dass es nicht zur Selbstzerstörung kommt. Dafür sei aber eine Abkehr vom ,traditionellen' Lernen erforderlich, in dem lediglich früheres Wissen weitergegeben werde. Dieses sei zu ersetzen durch ein Lernen, das in die Zukunft gerichtet sei und vor allem in der Bearbeitung von Problemen bestehen müsse, die die Menschheit insgesamt betreffen und zu neuen Wegen und Problemlösungen führt.

Wolfgang Klafki griff diese Vorstellungen in seinen didaktischen Veröffentlichungen wiederholt auf und forderte konsequent im Zusammenhang mit seinem aktualisierten Bildungsverständnis (KLAFKI 1991), dass das nach Unterrichtsfächern gegliederte schulische Curriculum ergänzt werden müsse durch fächerübergreifende und projektartig zu bearbeitende „epochaltypische Schlüsselprobleme“. $\mathrm{Zu}$ befürchten sei nämlich, dass uns alle angehende Probleme in einem nach Fächern organisierten Unterricht nicht bzw. nicht in der erforderlichen Komplexität berücksichtigt würden. Gemeint waren solche Probleme wie die Friedensfrage, die Umweltfrage, die gesellschaftlich produzierte Ungleichheit, die Gefahren und Möglichkeiten der neuen technischen Steuerungs-, Informations- und Kommunikationsmedien, die Subjektivität des einzelnen und das Phänomen der Ich-Du-Beziehung. Das sind fürwahr gewichtige Probleme, die uns alle in der jetzigen, aber ebenso in der absehbaren Zukunft betreffen, an deren Lösung wir, aktuell aber vor allem die neu heranwachsende Generation, zukünftig mitzuwirken haben.

Diese Ideen werden heute wieder erfreulich konsequent in der Bewegung „Fridays for Future" aufgegriffen und - aktuell mit dem Schwerpunkt Klimawandel - weitergeführt. Ihre Bearbeitung ist in einem traditionellen Fachunterricht kaum möglich.

Zur Realisierung von projektartigem Unterricht sind organisatorische Rahmenbedingungen etwa in der Stundenplangestaltung erforderlich und darüber hinaus unterrichtliche und methodische Kompetenzen bei Lehrer*innen und Schüler*innen. Die Lehrer*innen müssen letztlich in der Lage sein, die Lernenden dazu zu befähigen, an wünschenswerten Problemlösungen aktiv mitzuwirken. Angesprochen ist damit eine große Aufgabe, der sich auch die Lehrer*innenbildung stellen muss. Die Lernwerkstatt gewinnt insbesondere in diesem Zusammenhang ihre Bedeutsamkeit für die Sekundarstufen unserer Schulen. 


\section{Zur Entwicklung in Halle}

Vor diesem politisch-didaktischen Hintergrund setzte sich Meinert Meyer mit meiner Unterstützung, die wir nach der Wende nach Halle als Professoren für Allgemeine Didaktik berufen wurden, für die Einrichtung einer Hochschullernwerkstatt an der Martin-Luther-Universität Halle-Wittenberg ein. Unsere vor allem bildungstheoretisch begründete Überzeugung war es, dass Schule und Lehrer*innenbildung einen Beitrag leisten müssten zur Förderung selbstständigen Lernens und darüber hinaus für lebenslanges Lernen mit dem Ziel der Verwirklichung einer lebendigen Demokratie. Daher vertraten wir die Auffassung, dass im Curriculum der Lehrer*innenbildung für alle Schulstufen der Aufbau unterrichtsmethodischer Kompetenz ein wichtiger Bestandteil sein müsse und in diesem Zusammenhang auch die Anleitung zu eigenverantwortlichem bzw. selbstständigem Lernen.

Unser Interesse galt der Realisierung eines „schüleraktiven Unterrichts“, der wie Klafki forderte - Bildung in einem umfassenden demokratisch-emanzipatorischen Sinne ermöglicht, der zu Selbst- und Mitbestimmung sowie zu Solidarität befähigt. Beide brachten wir unsere bildungstheoretischen und didaktischen Überzeugungen in die Lehrer*innenbildung an der Universität in Halle und insbesondere Meinert Meyer - auch in die Diskussion um die Neugestaltung der Rahmenrichtlinien für Sekundarschulen und Gymnasien in Sachsen-Anhalt ein. Ab 1995 leitete ich ein Forschungsprojekt zur Begleitung der Einführung der Förderstufe und zur Einführung der stark reformpädagogisch geprägten Förderstufendidaktik.

Mit der Einführung der Förderstufe wurde in Sachsen-Anhalt Mitte der 1990er Jahre nicht nur eine schulstrukturelle Reform eingeleitet (Weiterführung des gemeinsamen Unterrichts für alle Schüler*innen im 5. und 6. Schuljahrgang im Rahmen der Sekundarschule), sondern auch eine anspruchsvolle innere Schulreform. Die zentralen Stichworte waren: längeres gemeinsames Lernen, Binnendifferenzierung im Fachunterricht, handlungsorientiertes und fächerübergreifendes Lernen, Projektarbeit und Erziehung zur Selbstständigkeit (Kultusministerium 1996).

In der Broschüre des Kultusministeriums zum Förderstufen-Projekt „Miteinander leben" heißt es z. B. interessanterweise:

„Zusammenfassend läßt sich sagen, dass das Ziel der Schulausbildung nicht nur in der Vermittlung von Wissen, sondern in einer umfassenden Förderung der Persönlichkeit zu sehen ist. In diesem Zusammenhang gewinnen zunehmend Bedeutung:

- die Fähigkeit zur Selbstständigkeit und zur Entfaltung von Eigeninitiativen, um Probleme eigenverantwortlich und kreativ lösen zu können; 
- die Fähigkeit zur Teamarbeit und damit einhergehend erhöhte kommunikative Kompetenz und Koordinationsfähigkeit;

- die Fähigkeit zu Planung und Kontrolle in Verbindung mit interdisziplinärem Denken,

- die Bereitschaft zu ständigem Lernen in einer beruflichen und gesellschaftlichen Umwelt, die sich in stetigem Wandel befindet; hierbei geht es nicht nur um veränderte Wissensstrukturen, sondern vor allem um Kenntnisse von Methoden und Lerntechniken;

- die Überprüfung bisheriger geschlechtsspezifischer Rollenklischees, verbunden mit dem Ziel, dass alle Berufsfelder gleichermaßen erreicht werden können.

Schule ist somit nicht nur Ausbildungsstätte, sondern auch eine wichtige Sozialisationsinstanz." (KULTUSMINISTERIUM 1997, 28).

Anmerken möchte ich hier noch, dass das Kultusministerium Sachsen-Anhalt in den "Grundsätzen und Anregungen für den Unterricht", die den Lehrkräften der Sekundarschulen mit der Einführung der Förderstufe an die Hand gegeben wurden, für die unterrichtliche Gestaltung der neu eingeführten Projektwochen das Buch von Karl FrEY (1982) empfohlen hat. Die ausgeführten Planungsbeispiele orientierten sich zudem an einem Planungsraster von Karl Frey.

Meinert Meyer beschrieb 1995 gemeinsam mit seinem Zwillingsbruder Hilbert Meyer in einem Beitrag (MeYER \& MeYER 1995) für den fortbildungsorientierten Sammelband Erziehungswissenschaft für Gymnasiallehrer*innen, dass zu einem „guten Unterricht“ neben einem „guten Frontalunterricht“ auch ein weit darüber hinausgehendes Methodenrepertoire der Lehrer*in erforderlich sei. Sie sahen sogar in der „Entfaltung des Methodenrepertoires im Unterricht“ den „erste[n] Schritt zur Schulreform" (ebd., 180).

Insoweit war der Einsatz für die Einrichtung einer Hochschullernwerkstatt für Studierende der Sekundarstufen verknüpft sowohl mit der allgemeindidaktischen und schulpädagogischen Diskussion der 1980er und 1990er Jahre ${ }^{2}$ als auch mit der Schul- und Curriculumreform nach der Wende in Sachsen-Anhalt in den 1990er Jahren.

Mit diesen gewichtigen Anstößen und ihrer theoretisch-didaktischen Absicherung, die zudem noch lerntheoretisch untersetzt wurden, war eine Grundlage für eine Arbeit in der Lernwerkstatt letztlich für alle Schulstufen geschaffen, mit der Argumentation der Gebrüder Meyer insbesondere auch für das Gymnasium.

Konkret hieß das in der Anfangszeit der Hochschullernwerkstatt am Institut für Pädagogik, dass entsprechend der Projektidee von John Dewey Fragen oder Problem- bzw. Aufgabenstellungen von studentischen Gruppen aufgenommen und bearbeitet wurden mit dem Ziel, am Ende des Semesters ein „Produkt“ vorzustel-

2 Vgl. hierzu auch Marotzki, Meyer \& Wenzel (1996). 


\section{Hartmut Wenzel}

len. Das Produkt sollte gewissermaßen den Ertrag der Recherchen präsentieren und damit kommunizierbar machen. Produkte konnten dabei ganz unterschiedliche Formen annehmen.

Einige Beispiele aus dem Wintersemester 1994/95 sind:

- eine Fotodokumentation zur Stadtentwicklung, in der historische Stadtansichten mit aktuellen Fotos kontrastiert werden,

- eine Mappe, in der mit Texten und Bildern die Entstehungsgeschichte eines „Fühlbuches" dokumentiert wird,

- Entwicklung und Erprobung einer Anleitung für ein Improvisationstheater,

- Erstellung eines Hörspiels,

- Bauen von Instrumenten und deren Vorstellung in einer „Musik-Session“.

Die Beispiele zeigen eine Vielfalt von unterschiedlichen Produkten, die im Zusammenhang mit der Arbeit in der Lernwerkstatt angestrebt wurden bzw. entstanden sind. Sie belegen zugleich, dass für die Realisierung und Präsentation der Projektergebnisse deutlich andere Räumlichkeiten und Ausstattungselemente erforderlich sind. Diese Erkenntnisse sind in die Planung der halleschen Hochschullernwerkstatt eingeflossen.

Mitte der 1990er Jahre wurden einige der Veranstaltungen in der Hochschullernwerkstatt ausgerichtet im Hinblick auf die Pädagogik der Förderstufe und daher auf handlungsorientiertes und projektartiges Lernen sowie auf die Gestaltung der Projektwochen für die 5. und 6. Klassen. Die leitende Idee war dabei, dass zukünftige Lehrkräfte besser auf solche Lernformen vorbereitet sind, wenn sie diese selbst praktisch erlebt haben und zwar von der kooperativen Planung über die Beund Erarbeitungsschritte bis hin zur möglichst schulöffentlichen Präsentation.

Um die Jahrtausendwende gelangten mit der UN-Dekade für nachhaltige Entwicklung die von Wolfgang Klafki propagierten „epochaltypischen Schlüsselprobleme" verstärkt in den Blick und wurden wiederholt in Seminaren in der Hochschullernwerkstatt in fächerübergreifenden Projekten bearbeitet. In einem dieser Seminare wählte ich als Ausgangspunkt für solche Projekte die Aufgabe, inwieweit das Thema „Brot" von den Studienfächern der Studierenden her bearbeitet werden könnte, in einem anderen das Thema „Wasser“. Es war eine bedeutsame Erfahrung, dass solche allgemeinen Themen relativ leicht anregend und ergiebig aus unterschiedlicher Fachperspektive bearbeitet werden können und wie dabei auch Aspekte der Globalisierung und Nachhaltigkeit in anspruchsvoller Weise in den Blick kommen können. Im Seminar zum Thema "Wasser" besuchten wir auch das hiesige Wasserwerk als Beispiel dafür, dass im Rahmen von Projekten außerschulische Lernorte einbezogen werden können. 


\section{Abschließende Bemerkungen}

Seit Einrichtung der Hochschullernwerkstatt am Institut für Pädagogik Mitte der 1990er Jahre gab es von den Erziehungswissenschaften her für viele Jahre ein kontinuierliches Angebot zu handlungsorientiertem, projektartigem Unterricht oder auch zu kooperativem Lernen, zur Entwicklung methodischer Kompetenz, zu Zukunftswerkstätten sowie zur Auseinandersetzung mit reformpädagogischen Ansätzen und damit zur als bedeutsam erachteten „Entfaltung des Methodenrepertoires".

Im Zusammenhang mit der Eröffnung der integrierten Hochschullernwerkstatt 2005 wurde das damals diskutierte Verständnis auf der Homepage folgendermaßen formuliert:

Was sind Lernwerkstätten?

Oberflächlich betrachtet sind es „Räume, die voller Material stecken und in denen sich Erwachsene, zum Teil mit Kindern treffen, um sich mit diesen Materialien auseinanderzusetzen - durch eigenes Tun und aktive Nutzung all dessen, was in den Räumen vorhanden ist, durch Sichten des Materials für eine spätere Verwendung in anderen Lernzusammenhängen oder durch Gespräche über pädagogische Fragen in Arbeits- bzw. Beratungssituationen" (ERNST \& WedEKInd 1990, 6).

Blickt man auf die zuvor genannten Begründungsmuster für (Hochschul-)Lernwerkstätten auch für die Sekundarstufen, die maßgeblich für die Einrichtung der Hochschullernwerkstatt an der Martin-Luther-Universität in Halle waren, dann ist festzustellen, dass die angeführten Gründe auch heute noch oder wieder relevant sind.

Es ist daher zu wünschen, dass die Hochschullernwerkstatt auch für die Sekundarstufen in der zuvor ausgeführten Tradition und Zielsetzung fortgeführt wird.

\section{Literatur}

Bohnsack, Fritz; Bohnsack, Liselotte; Möller, Elisabeth; Schön, Hans; Schürmann, Gunna; Wenzel, Hartmut \& Wesemann, Matthias (1984): Schüleraktiver Unterricht. Möglichkeiten und Grenzen der Überwindung von „Schulmüdigkeit“ im Alltagsunterricht (Beltz Forschungsbericht). Weinheim und Basel: Beltz Verlag.

ERnst, Karin \& Wedekind, Hartmut (Hrsg.)(1993) Lernwerkstätten in der Bundesrepublik Deutschland und Österreich: eine Dokumentation. Bad Aurach.

Frey, Karl (1982): Die Projektmethode: „der Weg zum bildenden Tun“. Weinheim: Beltz Verlag.

Gudjons, Herbert (1987): Handlungsorientiert lehren und lernen: Schüleraktivierung - Selbsttätigkeit - Projektarbeit. Bad Heilbrunn: Klinkhardt.

KLAFKI, Wolfgang (1991): Grundzüge eines neuen Allgemeinbildungskonzeptes, im Zentrum: Epochaltypische Schlüsselprobleme. In: KLAfKI, Wolfgang: Neue Studien zur Bildungstheorie und Didaktik. Zeitgemäße Allgemeinbildung und kritisch-konstruktive Didaktik. Weinheim: Beltz Verlag, 43-81.

Kultusministerium des Landes Sachsen-Anhalt (Hrsg.) (1996): Grundlagen der Förderstufe. Magdeburg. 


\section{Hartmut Wenzel}

Kultusministerium des Landes Sachsen-Anhalt (Hrsg.)(1997): Miteinander leben. Grundsätze und Anregungen zum Unterricht. Förderstufe Schuljahrgang 5, Pflichtprojekt 1. Magdeburg.

Marotzki, Winfried; Meyer, Meinert \& Wenzel, Hartmut (Hrsg.) (1996): Erziehungswissenschaft für Gymnasiallehrer. Weinheim: Deutscher Studien Verlag.

Meadows, Dennis (1972): Die Grenzen des Wachstums. Bericht zur Lage der Menschheit. Stuttgart: Deutsche Verlagsanstalt.

Meyer, Hilbert \& Meyer, Meinert (1996): Frontalunterricht und anderes - Versuch einer Unterrichtsmethodik für das Gymnasium. In: MarotzKi, Winfried; Meyer, Meinert \& Wenzel, Hartmut (Hrsg.) (1996): Erziehungswissenschaft für Gymnasiallehrer. Weinheim: Deutscher Studien Verlag, 178-216.

PecceI, Aurelio (Hrsg.) (1980): Zukunftschance Lernen. Bericht für die achtziger Jahre. Berlin: Goldmann Verlag.

WenZel, Hartmut (1987): Unterricht und Schüleraktivität. Probleme und Möglichkeiten der Entwicklung von Selbststeuerungsfähigkeiten im Unterricht. Weinheim: Deutscher Studien Verlag.

Wenzel, Hartmut; Wesemann, Matthias \& Bohnsack, Fritz (Hrsg.) (1990): Schulinterne Lehrerfortbildung. Ihr Beitrag zu schulischer Selbstentwicklung. Weinheim: Beltz Verlag. 